\title{
Painlevé Functions in Statistical Physics
}

\author{
by
}

Craig A. TRACY and Harold Widom

\begin{abstract}
We review recent progress in limit laws for the one-dimensional asymmetric simple exclusion process (ASEP) on the integer lattice. The limit laws are expressed in terms of a certain Painlevé II function. Furthermore, we take this opportunity to give a brief survey of the appearance of Painlevé functions in statistical physics.
\end{abstract}

2010 Mathematics Subject Classification: 34M55, 60K35, 82B23.

Keywords: Painlevé function, ASEP, Bethe Ansatz, KPZ scaling.

"It was a pleasant surprise to me that such special functions actually appeared in concrete problems of theoretical physics..."

Mikio Sato 4

\section{§1. Introduction}

The appearance of Painlevé functions in the 2D Ising model is well-known [37, 64. Equally well-known is that this provided one impetus for M. Sato, T. Miwa and M. Jimbo [48 to develop their theory of holonomic quantum fields which connects the theory of isomondromy preserving deformations of linear differential equations with the $n$-point correlation functions of the $2 \mathrm{D}$ Ising model ${ }^{1}$

The general consensus in the field of "exactly solvable models" is that correlation functions are expressible in terms of Painlevé functions only in models that are free fermion models. More precisely, one expects that for the appearance of functions of the Painleve type, it is necessary for the underlying model or process to be a determinantal process in the sense of Soshnikov [52. In addition to the 2D

This is a contribution to the special issue "The golden jubilee of algebraic analysis".

Communicated by M. Kashiwara. Received December 13, 2009.

C. A. Tracy: Department of Mathematics, University of California, Davis, CA 95616, USA;

e-mail: tracy@math.ucdavis.edu

H. Widom: Department of Mathematics, University of California, Santa Cruz, CA 95064, USA; e-mail: widom@ucsc.edu 39.

${ }^{1}$ A complete account of the SMJ theory can be found in the recent monograph by Palmer

(C) 2011 Research Institute for Mathematical Sciences, Kyoto University. All rights reserved. 
Ising model, some notable examples where Painlevé functions arise in correlation functions include the one-dimensional impenetrable Bose gas [21, 28, 33, 34, the Ising chain in a tranverse field [41], the distribution functions of random matrix theory [1, 5, 16, 22, 28, 56, 57, 58, Hammersley's growth process 17, 8, corner and polynuclear growth models [9, 24, 29, 42, 43, and the totally asymmetric simple exclusion process (TASEP) [12, 29, 44]. Universality theorems in random matrix theory have extended the appearance of Painlevé functions to a wide class of matrix ensembles [13, 17, 18, 19, 51, 2] In recent developments [3, 45, 46, 47] Painlevé II appears in the long time asymptotics of explicit formulas for the exact height distribution for the KPZ equation [32] with narrow wedge initial condition.

As just noted, one does not expect Painlevé functions to arise in correlation functions in models that are exactly solvable in the sense of Baxter [1] but are not free fermion models, e.g. 6-vertex model, XXZ quantum spin chain, Baxter's 8-vertex model. Having said that, the universality conjecture arising in the theory of phase transitions suggests, for instance, that the scaling limit of a large class of ferromagnetic 2D Ising models is the same as that of the Onsager 2D Ising model; and hence, Painlevé functions are conjectured to appear (in the massive scaling limit) in models outside of the class of exactly solvable models. This last statement is substantiated by the developments in [3, 45, 46, 47.

In this paper we review recent progress [59, 60, 61, 62, 63] on the current fluctuations in the asymmetric simple exclusion process (ASEP) on the integer lattice $\mathbb{Z}[35,36]$. ASEP is in the class of Bethe Ansatz solvable models [23, 25] but only for certain values of the parameters is ASEP a determinantal process [29, 44, 49. That ASEP is Bethe Ansatz solvable comes as no surprise once one realizes that the generator of ASEP is a similarity (not unitary!) transformation of the XXZ-quantum spin Hamiltonian [2, 50, 65. Our main results relate the limiting current fluctuations in ASEP for certain initial conditions to the TW distributions $F_{1}$ and $F_{2}$ of random matrix theory [58, 59]. Both $F_{1}$ and $F_{2}$ are expressible in terms of the same Hastings-McLeod solution of Painlevé II [20, 26] (see $\S 4.2$ ).

\section{§2. Master equation and Bethe Ansatz solution}

Since its introduction in 1970 by F. Spitzer [53, the asymmetric simple exclusion process (ASEP) has attracted considerable attention both in the mathematics and physics literature due to the fact it is one of the simplest lattice models describing

\footnotetext{
${ }^{2}$ It is also worth noting that due to the close connection of random matrix theory to multivariate statistical analysis, these same distribution functions involving Painlevé functions are now routinely used in data analysis 30, 31, 40.
} 
transport far from equilibrium. Recall 35, 36, that the ASEP on the integer lattice $\mathbb{Z}$ is a continuous time Markov process $\eta_{t}$ where $\eta_{t}(x)=1$ if $x \in \mathbb{Z}$ is occupied at time $t$, and $\eta_{t}(x)=0$ if $x$ is vacant at time $t$. Particles move on $\mathbb{Z}$ according to two rules: (1) A particle at $x$ waits an exponential time with parameter one, and then chooses $y$ with probability $p(x, y)$; (2) If $y$ is vacant at that time it moves to $y$, while if $y$ is occupied it remains at $x$. The adjective "simple" refers to the fact that the allowed jumps are only one step to the right, $p(x, x+1)=p$, or one step to the left, $p(x, x-1)=q=1-p$. The totally asymmetric simple exclusion process (TASEP) allows jumps only to the right $(p=1)$ or only to the left $(p=0){ }^{3}$ In the mapping from the XXZ quantum spin chain, the anisotropy parameter $\Delta$ of the spin chain is related to the hopping probabilities $p$ and $q$ by

$$
\Delta=\frac{1}{2 \sqrt{p q}} \geq 1
$$

the ferromagnetic regime of the XXZ spin chain.

We begin with a system of $N$ particles and later take the limit $N \rightarrow \infty$. A configuration is specified by giving the location of the $N$ particles. We denote by $Y=\left\{y_{1}, \ldots, y_{N}\right\}$ with $y_{1}<\cdots<y_{N}$ the initial configuration of the process and write $X=\left\{x_{1}, \ldots, x_{N}\right\} \in \mathbb{Z}^{N}$. When $x_{1}<\cdots<x_{N}$ then $X$ represents a possible configuration of the system at a later time $t$. We denote by $P_{Y}(X ; t)$ the probability that the system is in configuration $X$ at time $t$, given that it was initially in configuration $Y$.

Given $X=\left\{x_{1}, \ldots, x_{N}\right\} \in \mathbb{Z}^{N}$ we set

$$
\begin{aligned}
& X_{i}^{+}=\left\{x_{1}, \ldots, x_{i-1}, x_{i}+1, x_{i+1}, \ldots, x_{N}\right\}, \\
& X_{i}^{-}=\left\{x_{1}, \ldots, x_{i-1}, x_{i}-1, x_{i+1}, \ldots, x_{N}\right\} .
\end{aligned}
$$

The master equation for a function $u$ on $\mathbb{Z}^{N} \times \mathbb{R}^{+}$is

$$
\frac{d}{d t} u(X ; t)=\sum_{i=1}^{N}\left(p u\left(X_{i}^{-} ; t\right)+q u\left(X_{i}^{+} ; t\right)-u(X ; t)\right),
$$

and the boundary conditions are, for $i=1, \ldots, N-1$,

$$
\begin{aligned}
& u\left(x_{1}, \ldots, x_{i}, x_{i}+1, \ldots, x_{N} ; t\right) \\
& \quad=p u\left(x_{1}, \ldots, x_{i}, x_{i}, \ldots, x_{N} ; t\right)+q u\left(x_{1}, \ldots, x_{i}+1, x_{i}+1, \ldots, x_{N} ; t\right) .
\end{aligned}
$$

The initial condition is

$$
u(X ; 0)=\delta_{Y}(X) \quad \text { when } x_{1}<\cdots<x_{N} .
$$

\footnotetext{
${ }^{3}$ It is TASEP that is a determinantal process.
} 
The basic fact is that if $u(X ; t)$ satisfies the master equation, the boundary conditions, and the initial condition, then $P_{Y}(X ; t)=u(X ; t)$ when $x_{1}<\cdots<x_{N}$. This is, of course, one of Bethe's basic ideas (see, e.g., [10]): incorporate the interaction (in this case the exclusion property) into the boundary conditions (2) of a free particle system (1).

Recall that an inversion in a permutation $\sigma$ is an ordered pair $\{\sigma(i), \sigma(j)\}$ in which $i<j$ and $\sigma(i)>\sigma(j)$. We define [65]

$$
S_{\alpha \beta}=-\frac{p+q \xi_{\alpha} \xi_{\beta}-\xi_{\alpha}}{p+q \xi_{\alpha} \xi_{\beta}-\xi_{\beta}}
$$

and then

$$
A_{\sigma}=\prod\left\{S_{\alpha \beta}:\{\alpha, \beta\} \text { is an inversion in } \sigma\right\} .
$$

We also set

$$
\varepsilon(\xi)=p \xi^{-1}+q \xi-1 .
$$

In the next theorem we shall assume $p \neq 0$, so the $A_{\sigma}$ are analytic at zero in all the variables. Here and later all differentials $d \xi$ incorporate the factor $(2 \pi i)^{-1}$.

Theorem 2.1. We have

$$
P_{Y}(X ; t)=\sum_{\sigma \in \mathcal{S}_{N}} \int_{\mathcal{C}_{r}} \cdots \int_{\mathcal{C}_{r}} A_{\sigma} \prod_{i} \xi_{\sigma(i)}^{x_{i}-y_{\sigma(i)}-1} e^{\sum_{i} \varepsilon\left(\xi_{i}\right) t} d \xi_{1} \cdots d \xi_{N},
$$

where $\mathcal{C}_{r}$ is a circle centered at zero with radius $r$ so small that all the poles of the integrand lie outside $\mathcal{C}_{r}$.

The proof that $P_{Y}(X ; t)$ satisfies (1) is immediate and the fact it satisfies the boundary conditions (2) is exactly the same argument as in the XXZ problem [65. The difficulty lies in showing (5) satisfies the initial condition (3). Observe that the term in (5) corresponding to the identity permutation does satisfy the initial condition. Thus the proof will be complete once one demonstrates that the remaining $n !-1$ terms sum to zero at $t=0$. This is indeed the case and the result depends crucially upon the choice of the contours $\mathcal{C}_{r}$ [59]. For the special case of TASEP, $p=1$, it follows from (4) and (5) that the right-hand side of (5) can be expressed as an $N \times N$ determinant as first obtained in [49].

We note that unlike the usual applications of Bethe Ansatz, it is not the spectral theory of the operator that is of interest but rather the transition probability $P_{Y}(X ; t)$. Thus there are no Bethe equations in our approach; and hence, no issues concerning the completeness of the Bethe eigenfunctions. Indeed, there is not even an Ansatz in this approach! We remark that this result extends with only minor modifications to the solution $\Psi\left(x_{1}, \ldots, x_{N} ; t\right)$ of the time-dependent Schrödinger 
equation with XXZ Hamiltonian where the $x_{i}$ 's denote the location of the $N$ "up spins" in a sea of "down spins" on $\mathbb{Z}$.

\section{§3. Marginal distributions and the large $N$ limit}

We henceforth assume $q>p$ so there is a net drift of particles to the left. Here we consider two different initial conditions. The first, called step initial condition, starts with particles located at $\mathbb{Z}^{+}=\{1,2, \ldots\}$. The second initial condition is the step Bernoulli initial condition: each site in $\mathbb{Z}^{+}$, independently of the others, is initially occupied with probability $\rho, 0<\rho \leq 1$; all other sites are initially unoccupied. In each of these cases it makes sense to speak of the position of the $m$ th particle from the left at time $t, x_{m}(t)$, and its distribution function $\mathbb{P}\left(x_{m}(t) \leq x\right)$. It is elementary to relate $\mathbb{P}\left(x_{m}(t) \leq x\right)$ to the distribution of the total current $\mathcal{T}$ at position $x$ at time $t$,

$$
\mathcal{T}(x, t):=\text { number of particles } \leq x \text { at time } t \text {; }
$$

namely,

$$
\mathbb{P}(\mathcal{T}(x, t) \leq m)=1-\mathbb{P}\left(x_{m+1}(t) \leq x\right) .
$$

For this reason we first concentrate on $\mathbb{P}_{Y}\left(x_{m}(t) \leq x\right)$ and only at the end translate the results into statements concerning $\mathcal{T}$. (The subscript $Y$ denotes the initial configuration.)

Now for finite $Y$,

$$
\begin{aligned}
& \mathbb{P}_{Y}\left(x_{m}(t)=x\right) \\
& \quad=\sum_{x_{1}<\cdots<x_{m-1}<x<x_{m+1}<\cdots<x_{N}} P_{Y}\left(x_{1}, \ldots, x_{m-1}, x, x_{m+1}, \ldots, x_{N} ; t\right) .
\end{aligned}
$$

Since the contours $\mathcal{C}_{r}$ in (5) have $r \ll 1$, the sums over $x_{m+1}, \ldots, x_{N}$ can be interchanged with the integrations in variables $\xi_{\sigma(j)}^{x_{j}}, m+1 \leq j \leq N$, and the geometric series summed. To perform the sums over $x_{1}, \ldots, x_{m-1}$, the contours in the $\xi_{\sigma(j)}^{x_{j}}$ variables, $1 \leq j \leq m-1$, must be deformed out beyond the unit circle and then the sums can be interchanged with the integrations. This deformation beyond the unit circle can be done in such a way as not to encounter any poles of the integrand. However, upon deforming these contours back to $\mathcal{C}_{r}$ (after the geometric series are summed) one does encounter poles; and one finds some remarkable cancellations: only the residues from the poles at $\xi_{i}=1$ are nonzero. The result is a sum over all subsets of $S$ of $\{1, \ldots, N\}$ with $\left|S^{c}\right|<m$ whose summands involve $|S|$-dimensional 
integrals with contours $\mathcal{C}_{r} \cdot 4^{4}$ However, this resulting expression for $\mathbb{P}_{Y}\left(x_{m}(t)=x\right)$ is not so useful for taking the $N \rightarrow \infty$ limit.

The next step is to expand the contours to $\mathcal{C}_{R}, R \gg 1$. It is then possible to take the $N \rightarrow \infty$ limit in the resulting expressions. The details [59] are involved and they depend crucially upon some algebraic identities which we now state.

\section{§3.1. Three identities}

Let

$$
f(i, j):=p+q \xi_{i} \xi_{j}-\xi_{i}
$$

Identity \#1:

$$
\begin{array}{r}
\sum_{\sigma \in \mathcal{S}_{N}} \operatorname{sgn}(\sigma) \frac{\prod_{i<j} f(\sigma(i), \sigma(j))}{\left(\xi_{\sigma(1)}-1\right)\left(\xi_{\sigma(1)} \xi_{\sigma(2)}-1\right) \cdots\left(\xi_{\sigma(1)} \xi_{\sigma(2)} \cdots \xi_{\sigma(N)}-1\right)} \\
=q^{N(N-1) / 2} \frac{\prod_{i<j}\left(\xi_{j}-\xi_{i}\right)}{\prod_{j}\left(\xi_{j}-1\right)} .
\end{array}
$$

Identity $\# 2$ : For $N \geq m+1$,

$$
\sum_{|S|=m} \prod_{\substack{i \in S^{c} \\
j \in S^{c}}} \frac{f(i, j)}{\xi_{j}-\xi_{i}}\left(1-\prod_{j \in S^{c}} \xi_{j}\right)=q^{m}\left[\begin{array}{c}
N-1 \\
m
\end{array}\right]\left(1-\prod_{j=1}^{N} \xi_{j}\right)
$$

In (7) the sum runs over all subsets $S$ of $\{1, \ldots, N\}$ with cardinality $m$, and $S^{c}$ denotes the complement of $S$ in $\{1, \ldots, N\}$. Here $\left[\begin{array}{l}N \\ m\end{array}\right]$ is a slightly modified $\tau$-binomial coefficient, $\tau:=p / q$,

$$
\begin{aligned}
{[N] } & :=\frac{p^{N}-q^{N}}{p-q}, \quad[0]:=1, \\
{[N] ! } & :=[N][N-1] \cdots[1], \\
{\left[\begin{array}{l}
N \\
m
\end{array}\right] } & :=\frac{[N] !}{[m] ![N-m] !}=q^{m(N-m)}\left[\begin{array}{l}
N \\
m
\end{array}\right]_{\tau}
\end{aligned}
$$

where $\left[\begin{array}{c}N \\ m\end{array}\right]_{\tau}$ is the usual $\tau$-binomial coefficient. We define $\left[\begin{array}{c}N \\ m\end{array}\right]_{\tau}=0$ for $m<0$. In proving (7) we first proved a simpler identity:

Identity \#3:

$$
\sum_{|S|=m} \prod_{\substack{i \in S \\
j \in S^{c}}} \frac{f(i, j)}{\xi_{j}-\xi_{i}}=\left[\begin{array}{l}
N \\
m
\end{array}\right]
$$

We believe that these identities suggest a deeper mathematical structure that is yet to be discovered.

\footnotetext{
${ }^{4}$ This is Theorem 5.1 in 59 .
} 


\section{§3.2. Final expression for $\mathbb{P}\left(x_{m}(t) \leq x\right)$ for step Bernoulli initial conditions}

We denote by $\mathbb{P}_{\rho}$ the probability measure for ASEP with step Bernoulli initial conditions. For $\rho=1$ the measure is ASEP with step initial condition. Let

$$
c_{m, k}:=(-1)^{m} q^{k(k-1)} \tau^{m(m-1) / 2} \tau^{-k m}\left[\begin{array}{c}
k-1 \\
m-1
\end{array}\right]_{\tau} .
$$

Observe that $c_{m, k}=0$ when $m>k$.

Theorem 3.1 ([59, 63]). Assume $q>p$. Then

$$
\begin{aligned}
\mathbb{P}_{\rho}\left(x_{m}(t) \leq x\right)= & \sum_{k \geq 1} \frac{q^{k(k-1) / 2} \tau^{k(k+1) / 2}}{k !} c_{m, k} \int_{\mathcal{C}_{R}} \cdots \int_{\mathcal{C}_{R}} \prod_{1 \leq i \neq j \leq m} \frac{\xi_{j}-\xi_{i}}{f(i, j)} \\
& \times \prod_{i} \frac{\rho}{\xi_{i}-1+\rho(1-\tau)} \prod_{i=1}^{m} \frac{\xi_{i}^{x} e^{t \varepsilon\left(\xi_{i}\right)}}{1-\xi_{i}} d \xi_{i} .
\end{aligned}
$$

The contour $\mathcal{C}_{R}$, a circle of radius $R \gg 1$ centered at the origin, is chosen so that all (finite) poles of the integrand lie inside the contour.

We remark that for TASEP, $p=0$, the above sum reduces to one term; and this term can be shown to be equal to an $m \times m$ determinant.

The final simplification results if we use the identity [60]

$$
\operatorname{det}\left(\frac{1}{f(i, j)}\right)_{1 \leq i, j \leq k}=(-1)^{k}(p q)^{k(k-1) / 2} \prod_{i \neq j} \frac{\xi_{j}-\xi_{i}}{f(i, j)} \prod_{i} \frac{1}{\left(1-\xi_{i}\right)\left(q \xi_{i}-p\right)}
$$

in (8) and recognize the summand, a $k$-dimensional integral, as the coefficient of $\lambda^{k}$ in the Fredholm expansion of $\operatorname{det}\left(I-\lambda K_{\rho}\right)$ where $K_{\rho}$ acts on functions on $\mathcal{C}_{R}$ by

$$
f(\xi) \mapsto \int_{\mathcal{C}_{R}} K_{\rho}\left(\xi, \xi^{\prime}\right) f\left(\xi^{\prime}\right) d \xi^{\prime}
$$

where

$$
K_{\rho}\left(\xi, \xi^{\prime}\right)=q \frac{\xi^{x} e^{t \varepsilon(\xi)}}{p+q \xi \xi^{\prime}-\xi} \frac{\rho(\xi-\tau)}{\xi-1+\rho(1-\tau)}, \quad \tau=\frac{p}{q} .
$$

Note that when $\rho=1$, the case of step initial condition, the last factor in $K_{\rho}\left(\xi, \xi^{\prime}\right)$ equals one.

Since the coefficient of $\lambda^{k}$ in the expansion of $\operatorname{det}\left(I-\lambda K_{\rho}\right)$ is equal to

$$
\frac{(-1)^{k}}{k !} \int \operatorname{det}\left(I-\lambda K_{\rho}\right) \frac{d \lambda}{\lambda^{k+1}}
$$

this fact together with the $\tau$-binomial theorem gives the final result for $\mathbb{P}_{\rho}\left(x_{m}(t)\right.$ $\leq x)$. 
Theorem 3.2 ([59, 63]). Let $\mathbb{P}_{\rho}$ denote the probability measure for ASEP with step Bernoulli initial condition with density $\rho$ and $x_{m}(t)$ denote the position of the $m$ th particle from the left at time $t$. Then

$$
\mathbb{P}_{\rho}\left(x_{m}(t) \leq x\right)=\int_{\mathcal{C}} \frac{\operatorname{det}\left(I-\lambda K_{\rho}\right)}{\prod_{j=0}^{m-1}\left(1-\lambda \tau^{j}\right)} \frac{d \lambda}{\lambda}
$$

where the contour $\mathcal{C}$ is a circle centered at the origin enclosing all the singularities at $\lambda=\tau^{-j}, 0 \leq j \leq m-1$ and $K_{\rho}$ is the integral operator whose kernel is given by $(9)$.

\section{§4. Limit theorems}

\section{§4.1. KPZ scaling}

The scaling limit that is of most interest is the KPZ scaling limit [32, 54]. In the terminology here this scaling limit is

$$
m \rightarrow \infty, \quad t \rightarrow \infty \quad \text { with } \sigma=m / t \leq 1 \text { fixed. }
$$

As we shall see, the limiting distribution will depend upon the relative sizes of $\sigma$ and $\rho^{2}$. For the moment we concentrate on the cases $0<\sigma<\rho^{2}$ and $\sigma=\rho^{2}$ with $0<\rho \leq 1$. As in any central limit theorem, to obtain a nontrivial limit the $x$ in $\mathbb{P}_{\rho}\left(x_{m}(t) \leq x\right)$ must also be scaled (this too is part of KPZ scaling). In anticipation of the theorem we set

$$
x:=c_{1} t+c_{2} t^{1 / 3} s
$$

where the $\frac{1}{3}$ is the famous KPZ universality exponent [32, 38, and

$$
c_{1}:=-1+2 \sqrt{\sigma}, \quad c_{2}:=\sigma^{-1 / 6}(1-\sqrt{\sigma})^{2 / 3} .
$$

The two distribution functions that arise in the KPZ scaling limit are defined in the next section.

\section{§4.2. Distributions $F_{1}$ and $F_{2}$}

The distributions $F_{1}$ and $F_{2}$ can be defined by either their Fredholm determinant representations or their representations in terms of a Painlevé II function. Here we take the latter route. Let $q$ denote the solution to the Painlevé II equation

$$
q^{\prime \prime}=x q+2 q^{3}
$$

satisfying

$$
q(x) \sim \operatorname{Ai}(x), \quad x \rightarrow \infty,
$$


where $\operatorname{Ai}(x)$ is the Airy function. That such a solution exists and is unique was proved by Hastings and McCleod 26, $:^{5}$ Then we have

$$
\begin{aligned}
& F_{2}(s)=\exp \left(-\int_{s}^{\infty}(x-s) q(x)^{2} d x\right), \\
& F_{1}(s)=\exp \left(-\frac{1}{2} \int_{s}^{\infty} q(x) d x\right) F_{2}(s)^{1 / 2} .
\end{aligned}
$$

The asymptotics of these distributions as $x \rightarrow \infty$ is straightforward given the large $x$ asymptotics of the Airy function; however, the complete asymptotic expansion as $x \rightarrow-\infty$ has only recently been given [6]. For high-accuracy numerical evaluation of $F_{1}$ and $F_{2}$, it turns out that it is better to start with their Fredholm determinant representations [15].

\section{§4.3. Limit laws}

The asymptotic analysis [61, 63] of the Fredholm determinant in the formula for $\mathbb{P}_{\rho}\left(x_{m}(t) \leq x\right)$ in $(10)$ required the development of new methods since the operator $K_{\rho}$ is not of the usual "integrable integral operator" form normally appearing in random matrix theory [14, 27, 57. The main point is that the kernel $K_{\rho}$ has the same Fredholm determinant as a sum of two kernels; one has large norm but fixed spectrum and its resolvent can be computed exactly, and the other is better behaved [61.

We now state the results of this asymptotic analysis.

Theorem 4.1 ([61, 63]). When $0 \leq p<q, \gamma:=q-p$,

$$
\begin{aligned}
& \lim _{t \rightarrow \infty} \mathbb{P}_{\rho}\left(\frac{x_{m}(t / \gamma)-c_{1} t}{c_{2} t^{1 / 3}} \leq s\right)=F_{2}(s) \quad \text { when } 0<\sigma<\rho^{2}, \\
& \lim _{t \rightarrow \infty} \mathbb{P}_{\rho}\left(\frac{x_{m}(t / \gamma)-c_{1} t}{c_{2} t^{1 / 3}} \leq s\right)=F_{1}(s)^{2} \quad \text { when } \sigma=\rho^{2}, \rho<1 .
\end{aligned}
$$

This theorem implies a limit law for the current fluctuations. Define

$$
v=x / t, \quad a_{1}=(1+v)^{2} / 4, \quad a_{2}=2^{-4 / 3}\left(1-v^{2}\right)^{2 / 3} .
$$

Theorem 4.2. When $0 \leq p<q, \gamma:=q-p$,

(15) $\lim _{t \rightarrow \infty} \mathbb{P}_{\rho}\left(\frac{\mathcal{T}(v t, t / \gamma)-a_{1} t}{a_{2} t^{1 / 3}} \leq s\right)=1-F_{2}(-s) \quad$ when $-1<v<2 \rho-1$,
(16) $\lim _{t \rightarrow \infty} \mathbb{P}_{\rho}\left(\frac{\mathcal{T}(v t, t / \gamma)-a_{1} t}{a_{2} t^{1 / 3}} \leq s\right)=1-F_{1}(-s)^{2} \quad$ when $v=2 \rho-1, \rho<1$.

\footnotetext{
$20]$.

${ }^{5} \mathrm{~A}$ modern account of Painlevé transcendents can be found in the monograph by Fokas et al.
} 
Table 1. The mean $\left(\mu_{\beta}\right)$, variance $\left(\sigma_{\beta}^{2}\right)$, skewness $\left(S_{\beta}\right)$ and kurtosis $\left(K_{\beta}\right)$ of $F_{\beta}$, $\beta=1,2$. The numbers are courtesy of F. Bornemann and M. Prähofer.

\begin{tabular}{ccccc}
\hline$\beta$ & $\mu_{\beta}$ & $\sigma_{\beta}^{2}$ & $S_{\beta}$ & $K_{\beta}$ \\
\hline 1 & -1.206533574582 & 1.607781034581 & 0.29346452408 & 0.1652429384 \\
2 & -1.771086807411 & 0.8131947928329 & 0.224084203610 & 0.0934480876 \\
\hline
\end{tabular}

For step initial condition with $0<\sigma<1$ the limit laws are 13 and 15 61, 62. When $\sigma>\rho^{2}$ (or $v>2 \rho-1$ ) the fluctuations are of order $t^{1 / 2}$ and the limiting distribution is Gaussian (see [63] for details).

For TASEP, $p=0$, with step initial condition the limit law 15 was first proved by Johansson [29]. For TASEP with step Bernoulli initial condition the limit laws 15 and 16 were conjectured by Prähofer and Spohn [44] and proved recently by Ben Arous and Corwin 12. The fact that these limit laws remain essentially identical (the only change is the factor $\gamma$ in the time slot) is a very strong statement of KPZ Universality. From the integrable systems perspective, these results are, to the best of the authors' knowledge, the first limit laws of Bethe Ansatz solvable models (outside the class of determinantal models) where the correlation functions are expressible in terms of Painlevé functions.

\section{§5. Conclusions}

Today Painlevé functions occur in many areas of theoretical statistical physics. In the case of KPZ fluctuations there are now experiments [38, 55] on stochastically growing interfaces where quantities such as the skewness and the kurtosis of $F_{\beta}$ (see Table 1), as well as the distribution functions themselves, are compared with experiment. In 55] K. Takeuchi and M. Sano conclude that their measurements "... have shown without fitting that the fluctuations of the cluster local radius asymptotically obey the Tracy-Widom distribution of the GUE random matrices."

\section{Acknowledgements}

This work was supported by the National Science Foundation under grants DMS0906387 (first author) and DMS-0854934 (second author).

Note added in proof. The proof of Theorem 2.1 of [59] contains an error in Lemma 2.2. See the arXiv reference, version 3, for a corrected proof. 


\section{References}

[1] M. Adler and P. van Moerbeke, Hermitian, symmetric and symplectic random ensembles: PDEs for the distribution of the spectrum, Ann. of Math. 153 (2001), 149-189. Zbl 1033.82005 MR 1826412

[2] F. C. Alcaraz, M. Droz, M. Henkel and V. Rittenberg, Reaction-diffusion processes, critical dynamics, and quantum spin chains, Ann. Phys. 230 (1994), 250-302. Zbl 0787.60103 MR 1268278

[3] G. Amir, I. Corwin and J. Quastel, Probability distribution of the free energy of the continuum directed random polymer in 1+1 dimensions, Comm. Pure Appl. Math. 64 (2011), 466-537.

[4] E. Andronikof, Interview with Mikio Sato, Notices Amer. Math. Soc. 54 (2007), $208-222$. Zbl 1142.01324 MR 2285125

[5] J. Baik, Painlevé expressions for LOE, LSE and interpolating ensembles, Int. Math. Res. Notices 2002, no. 33, 1739-1789. Zbl 1016.60018 MR 1913947

[6] J. Baik, R. Buckingham and J. DiFranco, Asymptotics of the Tracy-Widom distributions and the total integral of a Painlevé II function, Comm. Math. Phys. 280 (2008), 463-497. Zbl pre05306099 MR 2395479

[7] J. Baik, P. Deift and K. Johansson, On the distribution of the length of the longest increasing subsequence of random permutations, J. Amer. Math. Soc. 12 (1999), 1119-1178. Zbl 0932.05001 MR 1682248

[8] J. Baik and E. M. Rains, Symmetrized random permutations, in Random matrices and their applications, MSRI Publ. 21, Cambridge Univ. Press, 2001, 1-19. Zbl 0989.60010 MR 1842780

[9] L Limiting distributions for a polynuclear growth model with external sources, J. Statist. Phys. 100 (2000), 523-541. Zbl 0976.82043 MR 1788477

[10] M. T. Batchelor, The Bethe ansatz after 75 years, Physics Today, January 2007, 36-40.

[11] R. J. Baxter, Exactly solved models in statistical mechanics, Academic Press, 1982. Zbl 0538.60093 MR 0690578

[12] G. Ben Arous and I. Corwin, Current fluctuations for TASEP: A proof of the PrähoferSpohn conjecture, Ann. Probab. 39 (2011), 104-138. Zbl pre05848966

[13] P. Bleher and A. R. Its, Semiclassical asymptotics of orthogonal polynomials, RiemannHilbert problem, and universality in the matrix model, Ann. of Math. 150 (1999), 185-266. Zbl 0956.42014 MR 1715324

[14] G. Blower, Random matrices: high dimensional phenomena, London Math. Soc. Lecture Note Ser. 367, Cambridge Univ. Press, 2009. Zbl pre05625880 MR 2566878

[15] F. Bornemann, On the numerical evaluation of distributions in random matrix theory: A review with an invitation to experimental mathematics, Markov Process. Related Fields 16 (2010), 803-866.

[16] A. Borodin and P. Deift, Fredholm determinants, Jimbo-Miwa-Ueno $\tau$-functions, and representation theory, Comm. Pure Appl. Math. 55 (2002), 1160-1230. Zbl 1033.34089 MR 1908746

[17] P. Deift and D. Gioev, Universality at the edge of the spectrum for unitary, orthogonal and symplectic ensembles of random matrices, Comm. Pure Appl. Math. 60 (2007), 867-910. Zbl 1119.15022 MR 2306224

[18] Random matrix theory: invariant ensembles and universality, Courant Lecture Notes in Math. 18, Courant Inst. Math. Sci., New York, Amer. Math. Soc., Providence, RI, 2009. Zbl 1171.15023 MR 2514781 
[19] P. Deift, T. Kriecherbauer, T-R. McLaughlin, S. Venakides and X. Zhou, Uniform asymptotics for polynomials orthogonal with respect to varying exponential weight and applications to universality questions in random matrix theory, Comm. Pure Appl. Math. 52 (1999), 1335-1425. Zbl 0944.42013 MR 1702716

[20] A. S. Fokas, A. R. Its, A. A. Kapaev and V. Yu. Novokshenov, Painlevé transcendents: the Riemann-Hilbert approach, Amer. Math. Soc., 2006. Zbl 1111.34001||MR 2264522

[21] P. J. Forrester, N. E. Frankel, T. M. Garoni and N. S. Witte, Painlevé transcendent evaluations of finite system density matrices for 1d impenetrable bosons, Comm. Math. Phys. 238 (2003), 257-285. Zbl 1041.82003 MR 1990877

[22] P. J. Forrester and N. S. Witte, Random matrix theory and the sixth Painlevé transcendent, J. Phys. A 39 (2006), 12211-12233. Zbl 1119.34070 MR 2266222

[23] J. de Gier and F. H. L. Essler, Exact spectral gaps of the asymmetric exclusion process with open boundaries, J. Statist. Mech. 2006, P12011.

[24] J. Gravner, C. A. Tracy and H. Widom, Limit theorems for height fluctuations in a class of discrete space and time growth models, J. Statist. Phys. 102 (2001), 1085-1132. Zbl 0989.82030 MR 1830441

[25] L.-H. Gwa and H. Spohn, Bethe solution for the dynamical-scaling exponent of the noisy Burgers equation, Phys. Rev. A 46 (1992), 844-854.

[26] P. Hastings and J. B. McLeod, A boundary value problem associated with the second Painlevé transcendent and the Korteweg-de Vries equation, Arch. Ration. Mech. Anal. 73 (1980), 31-51. Zbl 0426.34019 MR 0555581

[27] A. R. Its, A. G. Izergin, V. E. Korepin and N. A. Slavnov, Differential equations for quantum correlation functions. Int. J. Modern Phys. B 4 (1990), 1003-1037. Zbl 0719.35091 MR 1064758

[28] M. Jimbo, T. Miwa, Y. Môri and M. Sato, Density matrix of an impenetrable Bose gas and the fifth Painlevé transcendent, Phys. D 1 (1980), 80-158. Zbl 1194.82007 MR 0573370

[29] K. Johansson, Shape fluctuations and random matrices, Comm. Math. Phys. 209 (2000), 437-476. Zbl 0969.15008 MR 1737991

[30] I. M. Johnstone, On the distribution of the largest principal component, Ann. Statist. 29 (2001), 295-327. Zbl 1016.62078 MR 1863961

[31] _ Multivariate analysis and Jacobi ensembles: largest eigenvalue, Tracy-Widom limits and rates of convergence, Ann. Statist. 36 (2008), 2638-2716. Zbl pre05503373 MR 2485010

[32] M. Kardar, G. Parisi and Y.-C. Zhang, Dynamic scaling of growing interfaces, Phys. Rev. Lett. 56 (1986), 889-892. Zbl 1101.82329

[33] T. Kojima, Ground-state correlation functions for an impenetrable Bose gas with Neumann or Dirichlet boundary conditions, J. Statist. Phys. 88 (1997), 713-743. Zbl 0945.82513 MR 1467627

[34] V. E. Korepin, N. M. Bogoliubov and A. G. Izergin, Quantum inverse scattering method and correlation functions, Cambridge Univ. Press, 1993. Zbl 0787.47006 MR 1245942

[35] T. M. Liggett, Interacting particle systems, Springer, Berlin, 2005 [Reprint of the 1985 edition]. Zbl 1103.82016 MR 0776231

[36] _ Stochastic interacting systems: contact, voter and exclusion processes, Springer, Berlin, 1999. Zbl 0949.60006 MR 1717346

[37] B. M. McCoy, C. A. Tracy and T. T. Wu, Painlevé functions of the third kind, J. Math. Phys. 18 (1977), 1058-1092. Zbl 0353.33008 MR 0473322

[38] L. Miettinen, M. Myllys, J. Merikoski and J. Timonen, Experimental determinination of the KPZ height-fluctuation distributions, Eur. Phys. J. B 46 (2005), 55-60. 
[39] J. Palmer, Planar Ising correlations, Birkhäuser Boston, 2007. Zbl 1136.82001 MR 2332010

[40] N. Patterson, A. L. Price and D. Reich, Population structure and eigenanalysis, PLOS Genetics 2 (2006), 2074-2093.

[41] J. H. H. Perk, H. W. Capel G. R. W. Quispel and F. W. Nijhoff, Finite-temperature correlations for the Ising chain in a transverse field, Phys. A 123 (1984), 1-49. MR 0736481

[42] M. Prähofer and H. Spohn, Universal distributions for growth processes in $1+1$ dimensions and random matrices, Phys. Rev. Lett. 2000, 4882-4885.

[43] Scale invariance of the PNG droplet and the Airy process, J. Statist. Phys. 108 (2002), 1071-1106. Zbl 1025.82010 MR 1933446

[44] Current fluctuations in the totally asymmetric simple exclusion process, Progr. Probab. 51 (2002), 185-204. Zbl 1015.60093 MR 1901953

[45] T. Sasamoto and H. Spohn, The crossover regime for the weakly asymmetric simple exclusion process, J. Statist. Phys. 140 (2010), 209-231. Zbl 1197.82093 MR 2659278

[46] - One-dimensional Kardar-Parisi-Zhang equation: An exact solution and its universality, Phys. Rev. Lett. 104 (2010), 230602.

[47] Exact height distributions for the KPZ equation with narrow wedge initial condition, Nuclear Phys. B 834 (2010), 523-542. MR 2628936

[48] M. Sato, T. Miwa and M. Jimbo, Holonomic quantum fields, I-V, Publ. RIMS Kyoto Univ. 14 (1978), 223-267; 15 (1979), 201-278; 15 (1979), 577-629; 15 (1979), 871-972; 16 (1980) 531-584. Zbl 0479.35072 MR 0594916

[49] G. M. Schütz, Exact solution of the master equation for the asymmetric exclusion process, J. Statist. Phys. 88 (1997), 427-445. Zbl 0945.82508 MR 1468391

[50] Exactly solvable models for many-body systems far from equilibrium, in Phase transitions and critical phenomena, Vol. 19, C. Domb and J. Lebowitz (eds.), Academic Press, London, 2000, 1-251. MR 2033539

[51] A. Soshnikov, Universality at the edge of the spectrum in Wigner random matrices, Comm. Math. Phys. 207 (1999), 697-733. Zbl 1062.82502 MR 1727234

[52] _ Determinantal random fields, Russian Math. Surveys 55 (2000), 923-975. Zbl 0991.60038 MR 1799012

[53] F. Spitzer, Interaction of Markov processes, Adv. Math. 5 (1970), 246-290. Zbl 0312.60060 MR 0268959

[54] H. Spohn, Kardar-Parisi-Zhang equation in one dimension and line ensembles, Pramana 64 (2005), 847-857.

[55] K. A. Takeuchi and M. Sano, Growing interfaces of liquid crystal turbulence: Universal scaling and fluctuations, Phys. Rev. Lett. 104 (2010), 230601.

[56] C. A. Tracy and H. Widom, Level-spacing distribution and the Airy kernel, Comm. Math. Phys. 159 (1994), 151-174. Zbl 0789.35152 MR 1257246

[57] Fredholm determinants, differential equations and matrix models, Comm. Math. Phys. 163 (1994), 33-72. Zbl 0813.35110 MR 1277933

[58] - Orthogonal and symplectic matrix ensembles, Comm. Math. Phys. 177 (1996), 727-754. Zbl 0851.60101 MR 1385083

[59] Integral formulas for the asymmetric simple exclusion process, Comm. Math. Phys. 279 (2008), 815-844; Erratum, arXiv:0704.2633,v3. Zbl 1148.60080 MR 2386729

[60] A Fredholm determinant representation in ASEP, J. Statist. Phys. 132 (2008), 291-300. Zbl 1144.82045 MR 2415104 
[61] , Asymptotics in ASEP with step initial condition, Comm. Math. Phys. 290 (2009), 129-154. Zbl 1184.60036 MR 2520510

[62] _ Total current fluctuations in the asymmetric simple exclusion process, J. Math. Phys. 50 (2009), 095204. Zbl pre05772165 MR 2566884

[63] _ On ASEP with step Bernoulli initial condition, J. Statist. Phys. 137 (2009), 825838. Zbl 1188.82043 MR 2570751

[64] T. T. Wu, B. M. McCoy, C. A. Tracy and E. Barouch, Spin-spin correlation functions for the two-dimensional Ising model: Exact theory in the scaling regime, Phys. Rev. B 13 (1976), 316-374.

[65] C. N. Yang and C. P. Yang, One-dimensional chain of anisotropic spin-spin interactions. I. Proof of Bethe's hypothesis for ground state in a finite system, Phys. Rev. 150 (1966), 321-327. 